\title{
Association of Hypertension and $\beta$-Blocker Use with Depression during Pregnancy
}

\author{
Alicia M. McCarthy1,2,3*, Ying Zhou4, Marci Adams', Rita Elue', Nicole Diaz², Beth A. Plunkett1 \\ ${ }^{1}$ Department of Obstetrics and Gynecology, NorthShore University HealthSystem, Evanston, IL, USA \\ ${ }^{2}$ Department of Obstetrics and Gynecology, Pritzker School of Medicine, University of Chicago, Chicago, IL, USA \\ ${ }^{3}$ Department of Maternal Fetal Medicine, Kaiser Permanente Medical Center, Walnut Creek, CA, USA \\ ${ }^{4}$ Research Institute, NorthShore University HealthSystem, Evanston, IL, USA \\ Email: *alicia.m.mccarthy@kp.org
}

How to cite this paper: McCarthy, A.M., Zhou, Y., Adams, M., Elue, R., Diaz, N. and Plunkett, B.A. (2018) Association of Hypertension and $\beta$-Blocker Use with Depression during Pregnancy. Open Journal of Obstetrics and Gynecology, 8, 925-935. https://doi.org/10.4236/ojog.2018.811094

Received: July 25, 2018

Accepted: September 1, 2018

Published: September 4, 2018

Copyright $\odot 2018$ by authors and Scientific Research Publishing Inc. This work is licensed under the Creative Commons Attribution International License (CC BY 4.0).

http://creativecommons.org/licenses/by/4.0/ Open Access

\begin{abstract}
Objective: To evaluate the association between hypertension and $\beta$-blocker (BB) use and antepartum depression risk. Patients and Methods: We conducted a retrospective cohort study of women who delivered within our integrated health system between 2009 and 2015, and completed an Edinburgh Postnatal Depression Scale (EPDS) during pregnancy. Increased depression risk was defined as EPDS score $\geq 10$, or an affirmative answer to question ten, endorsing self-harm. Antepartum hypertension was determined by blood pressure measurements and provider ICD-9 codes. Regression analyses examined the independent associations of $\mathrm{BB}$ use and hypertension on antepartum depression risk. Results: Of 9192 deliveries during the study time frame, $5 \%$ were hypertensive. Within the hypertensive group, 103 (22\%) used a single agent BB (BB Group), 325 (68\%) required no antihypertensive medication (No-Med Group), and 48 (10\%) used a non-BB single agent or multi-agent therapy (All-Other Group). After adjusting for covariates, compared to normotensive pregnancies, antepartum hypertension was significantly associated with both EPDS score $\geq 10$ (adjusted odds ratio [aOR] 1.61, 95\% confidence interval [CI] $1.17-2.21$ ) and endorsement of self-harm (aOR 1.76, $95 \%$ CI 1.05 - 2.95). In further analyses of depression risk in hypertensive pregnancies, there was no difference between the BB Group and No-Med Group (EPDS score $\geq 10$, aOR 1.22, 95\% CI 0.56 - 2.63; self-harm, aOR 0.84, 95\% CI 0.32 - 2.21), or between the All-Other Group and No-Med Group (EPDS $\geq 10$, aOR 1.42, 95\% CI 0.57 - 3.54; self-harm, aOR 1.04, 95\% CI 0.29 3.74). Conclusion: Women with antepartum hypertension have increased risk for depression and thoughts of self-harm. $\beta$-Blocker use is not associated with further increased risk.
\end{abstract}

\section{Keywords}

$\beta$-Blocker, Depression, Hypertension, Labetalol, Pregnancy 


\section{Introduction}

Depression and hypertension constitute two major morbidities of pregnancy. Depression is the most frequent mood disorder in the general population, and the prevalence of depression increases during pregnancy, likely impacting more than $12 \%$ of women during the second and third trimesters [1] [2] [3]. Hypertension is estimated to impact up to $10 \%$ of pregnancies worldwide and is a major contributor to maternal mortality [4] [5]. In 2006, $8 \%$ of deliveries in the United States were affected by hypertensive disorders [6]. Previous studies have suggested interplay between these morbidities, such that a diagnosis of hypertension increases risk of developing depression, and vice versa [7] [8]. The association between anti-hypertensive medications and depression has also gained public health interest. Some research in non-obstetric populations suggests that $\beta$-blocker (BB) use may be associated with depression and depressive symptoms [9] [10] [11] [12].

Initial concern linking depression and beta-adrenergic antagonists was generated in 1967, whereupon Waal reported a particularly high incidence of depression in a cohort of patients using propranolol, a highly lipophilic beta-blocker (BB), for antiarrhythmic therapy [11]. Since this initial report, numerous studies have further investigated, with mixed results, the depressogenic effects of BBs. The existence of beta-adrenergic receptors within the brain and cross-talk between BBs and serotonergic receptors in the brain yields biologic plausibility to the relationship between depression and beta-blockers [13]. Importantly, while evidence linking individual BBs with depression is mixed, a majority of studies encompass elderly, predominately male patients with a history of heart disease.

$\beta$-Blockers include one class of antihypertensive medication used for the treatment of hypertension in pregnancy, with the mixed alpha and non-selective beta adrenergic antagonist labetalol considered a first-line agent for treating hypertension [5]. To our knowledge, no study has evaluated the associations between BB use and depression during pregnancy. Given the increased incidence of depression and hypertension in pregnancy, the morbidity these diagnoses pose to a woman and her pregnancy, and non-obstetric literature raising concern for a possible association between $\mathrm{BB}$ use and depression, we set out to explore this association in pregnancy. Herein, our hypothesis is that $\mathrm{BB}$ use and hypertension during pregnancy are independently associated with increased depression risk in pregnancy.

\section{Patients and Methods}

This retrospective cohort study includes patients who received antenatal care within the NorthShore University HealthSystem (NSUHS) and subsequently delivered at a NSUHS hospital between January 1, 2009, and March 31, 2015. All women of at least 14 years of age who completed an Edinburgh Postnatal Depression Scale (EPDS) questionnaire during pregnancy were included in the study. Women without antepartum data, including those who received antepartum 
care outside NSUHS, and those who did not complete an EPDS during pregnancy, were excluded from analysis. Analyses encompassed the antepartum time frame up to and including completion of EPDS.

The EPDS survey is part of routine prenatal care in many obstetric practices within our Health System and is typically administered in the late second trimester. This 10-question survey is validated for depression screening in pregnant and postpartum patients [3]. Each question is scored from 0 to 3, with a total possible score ranging from 0 to 30 . Questions assess feelings during the prior seven days. For this study, increased depression risk was defined as a score of 1 3 on question number ten, which assesses for self-harm, or a total score greater than or equal to 10, a cutoff chosen to achieve a balance of sensitivity and specificity [14] [15]. Study protocol was approved by the NorthShore University HealthSystem Institutional Review Board, and is in accordance with the ethical standards of the 1964 Helsinki declaration and its later amendments or comparable ethical standards. For this type of study formal consent is not required.

The primary independent variables in this study are BB use and hypertension diagnosis. Hypertension during pregnancy was determined by blood pressure criteria and physician International Classification of Diseases, 9th Revision, Clinical Modification (ICD-9-CM) codes. Women were deemed to have hypertension with systolic blood pressure greater than or equal to $140 \mathrm{mmHg}$ and/or diastolic blood pressure greater than or equal to $90 \mathrm{mmHg}$, on two occasions at least six hours apart during pregnancy. Women with hypertension were also identified via outpatient and inpatient physician ICD-9-CM codes for preeclampsia (642.4, 642.5, and 642.6), gestational hypertension (642.3 and 642.9), chronic hypertension $(642.0,642.1$, and 642.2) and superimposed preeclampsia (642.7). Preeclampsia is defined by new onset hypertension and either proteinuria or end-organ damage in a woman who was previously normotensive. Gestational hypertension defines new onset hypertension, without proteinuria, after 20 weeks gestation. Chronic hypertension during pregnancy is a term encompassing women with pre-existing hypertension prior to pregnancy or within the first 20 weeks of gestation. Superimposed preeclampsia occurs when a woman with chronic hypertension develops worsening hypertension, proteinuria, or features of end-organ damage [5].

Women diagnosed with these hypertensive disorders of pregnancy and those women meeting blood pressure criteria as outlined above were all included in the hypertensive group. The normotensive group comprised the remaining women. Medication use was determined by issued prescriptions. Hypertensive women with a prescription for a single-agent $\mathrm{BB}$ dated $\geq 7$ days prior to completing the EPDS survey were classified as the BB Group. This time interval was selected to encompass the 7-day period addressed in the EPDS. A single-agent BB script issued less than 7 days prior to EPDS was considered a non-exposure. Hypertensive women not requiring an anti-hypertensive agent were classified as the No-Med Group. All other hypertensive women, including those using alternate single-agents or multiple anti-hypertensive agents with or without a $\mathrm{BB}$, 
were classified as the All-Other Group. Alternate anti-hypertensive medications included calcium channel blockers, thiazide diuretics, and antiadrenergic agents. Treatment and medication choice was per provider discretion.

To determine the association between hypertension in pregnancy and depression risk, normotensive pregnancies were compared to hypertensive pregnancies. To assess the association between BB use and depression risk, the BB Group was compared to the No-Med Group, and the All-Other Group was compared to the No-Med Group. Evaluated co-existing medical conditions included diabetes, thyroid disorder, and renal disease, which were ascertained via ICD-9-CM codes. Medication use was determined by an active prescription at the time of EPDS assessment. Corticosteroids were included for evaluation as this class of medication may induce mood symptoms [16]. Both hypo- and hyperthyroidism can be associated with depression, thus thyroid medication use was included for analysis. Finally, anxiety and depression requiring medical management were assessed by evaluating for benzodiazepine and antidepressant use [16]. Lifetime antidepressant use was also evaluated as a marker of depression significant enough to require medication, and this was determined by an antidepressant prescription at any historical point prior to EPDS assessment. Body mass index (BMI) information was captured at the EPDS visit.

Continuous variables were summarized as mean \pm standard deviation. The normality assumption for continuous variables was assessed using the Shapiro-Wilk test. Continuous variables were compared by Kruskal-Wallis test or Analysis of Variance. Categorical variables were summarized as frequencies and percentages and compared by Chi-square test or exact Chi-square test. Multivariable logistic regression analysis was conducted to compare depression scores among groups, with adjustment of those confounders determined to be significant. A confounder was retained in the model if it changed regression coefficients by more than $15 \%$. Adjusted odds ratios (aOR) and $95 \%$ confidence intervals (CI) were reported for pair-wise group comparisons. P values were adjusted for multiple comparisons. Statistical analysis was performed on SAS 9.3 (Cary, NC) on a Windows platform. A P value $<0.05$ was considered statistically significant.

\section{Results}

During the study period, there were 14,492 deliveries within our integrated health system. Deliveries without an associated antepartum EPDS and those without antepartum data were excluded from analysis, leaving 9,192 pregnancies for analysis (Figure 1). Of these pregnancies, 95\% $(n=8716)$ had no documented hypertension, while $5 \%(n=476)$ were hypertensive. There was no difference between normotensive and hypertensive groups in gestational age at the time of EPDS assessment ( $25.7 \pm 4.6$ and $25.5 \pm 5.8$, respectively, $\mathrm{P}=0.153)$. Of the hypertensive group, $22 \%(\mathrm{n}=103)$ were prescribed a BB for blood pressure control at least one week prior to antepartum depression screening (Figure 1). Labetalol was the BB utilized for 94 of these women. 


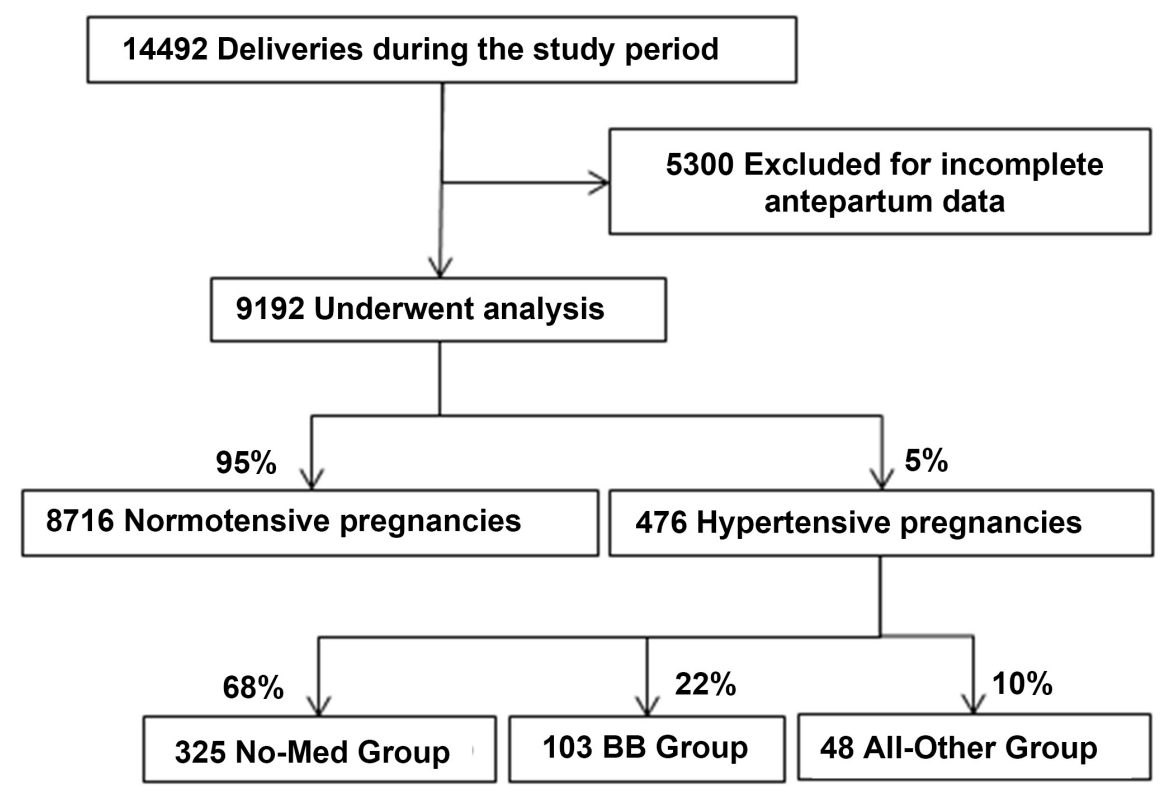

Figure 1. Analysis populations. Hypertensive women not requiring an anti-hypertensive agent were classified as the No-Med Group, those with a prescription for a single-agent $\mathrm{BB}$ dated $\geq 7$ days prior to completing the EPDS survey were classified as the BB Group, and those using alternate single-agents or multiple anti-hypertensive agents with or without a BB, were classified as the All-Other Group. BB: $\beta$-blocker.

Demographic subject characteristics are presented in Table 1. Women with hypertension were significantly older than the normotensive group, and they were more likely to be African American and publicly insured. Clinical characteristics are presented in Table 2. Hypertensive women were more likely than normotensive women to be obese, deliver prior to 37 weeks, smoke tobacco, and have concomitant chronic medical conditions (inclusive of diabetes, thyroid disorder, renal impairment). They were also more likely to be using an antidepressant at the time of EPDS.

We evaluated the association between antepartum hypertension and depression risk. In univariate analyses, compared to normotensive pregnancies, hypertensive pregnancies had significant increased risk of EPDS $\geq 10$ and endorsement of self-harm (Table 3). This significant association remained present in multivariate analyses. After adjustments, hypertensive pregnancies had significant increased risk of EPDS $\geq 10$ (aOR1.61, 95\% CI $1.17-2.21$ ), and significant increased risk of endorsing self-harm (aOR 1.76, 95\% CI 1.05 - 2.95).

We next evaluated the relation between $\mathrm{BB}$ use and depression risk. In pregnancies with hypertension, comparing the BB Group $(n=103)$ to the No-Med Group ( $\mathrm{n}=325$ ), there was no difference in EPDS $\geq 10$ or in thoughts of self-harm (Table 3). Furthermore, again among hypertensive pregnancies, comparing the No-Med Group $(n=325)$ to the All-Other Group $(n=48)$, there was also no difference in depression risk (Table 3). Thus, anti-hypertensive use in general, and particularly $\mathrm{BB}$ use, does not appear to be associated with depression risk in our cohort. 
Table 1. Patient sociodemographic characteristics.

\begin{tabular}{|c|c|c|c|c|c|}
\hline \multirow[t]{3}{*}{ Maternal Characteristic } & \multicolumn{5}{|c|}{ Hypertensive } \\
\hline & $\begin{array}{l}\text { Normotensive } \\
\quad(\mathrm{n}=8716)\end{array}$ & $\begin{array}{l}\text { No-Med } \\
(n=325)\end{array}$ & $\begin{array}{c}\text { BB } \\
(n=103)\end{array}$ & $\begin{array}{l}\text { All-Other } \\
(\mathrm{n}=48)\end{array}$ & $P$-value \\
\hline & Mean \pm SD & Mean \pm SD & Mean \pm SD & Mean \pm SD & \\
\hline \multirow[t]{2}{*}{ Age (y) } & $31.2 \pm 5.4$ & $31.6 \pm 5.8$ & $34.1 \pm 4.9$ & $36.2 \pm 5.4$ & $<0.001$ \\
\hline & $n(\%)$ & $n(\%)$ & $n(\%)$ & $n(\%)$ & \\
\hline Teen & $213(2)$ & $3(1)$ & $0(0)$ & $0(0)$ & 0.011 \\
\hline Race-ethnicity & & & & & $<0.001$ \\
\hline African American & $564(6)$ & $64(20)$ & $15(15)$ & $8(17)$ & \\
\hline Asian & $709(8)$ & $13(4)$ & $6(6)$ & $6(12)$ & \\
\hline Caucasian & $4447(51)$ & $139(43)$ & $56(54)$ & $20(42)$ & \\
\hline Hispanic & $1097(13)$ & $40(12)$ & $10(10)$ & $4(8)$ & \\
\hline Other & $1897(22)$ & $69(21)$ & $16(15)$ & $10(21)$ & \\
\hline Partner Status & & & & & 0.016 \\
\hline Partnered & $7072(82)$ & $241(74)$ & $85(83)$ & $36(75)$ & \\
\hline Unpartnered & $1574(18)$ & $81(25)$ & $18(17)$ & $10(21)$ & \\
\hline Health Insurance & & & & & $<0.001$ \\
\hline Private & $7024(82)$ & $237(73)$ & $79(77)$ & $35(73)$ & \\
\hline Public Aid & $1543(18)$ & $82(25)$ & $21(20)$ & $13(27)$ & \\
\hline
\end{tabular}

BB: $\beta$-blocker, SD: Standard Deviation.

\section{Discussion}

This retrospective study showed a significant association of hypertension during pregnancy and increased risk of depression and thoughts of self-harm. We found that BB use among those with hypertension is not associated with increased antepartum depression risk.

The association between hypertension and depression is supported by previous prospective studies in both obstetric and non-obstetric populations [7] [8] [17] [18]. While these studies are limited in evaluating specific cause and effect, various mechanisms have been proposed to explain the interplay between hypertension and depression. For example, depression increases sympathetic nervous system tone [19]. Additionally, numerous studies link inflammation to depression in non-pregnant adults [20] [21], yet pregnancy-specific studies are notably less consistent in the association between inflammatory markers and depression [22] [23]. Our findings further support the association between hypertension and depression in pregnancy, and they strengthen the need for further mechanistic studies.

With regards to depression and BB use, previous studies have both supported and refuted the depressogenic effects of BBs [9] [24]. The first report to raise concern over depression and BB use was published by Waal in 1967 [11]. This 
Table 2. Patient clinical characteristics.

\begin{tabular}{|c|c|c|c|c|c|}
\hline \multirow[t]{2}{*}{ Characteristic } & \multicolumn{5}{|c|}{ Hypertensive } \\
\hline & $\begin{array}{c}\text { Normotensive } \\
\begin{array}{c}(n=8716) \\
N(\%)\end{array}\end{array}$ & $\begin{array}{c}\text { No-Med } \\
(n=325) \\
N(\%)\end{array}$ & $\begin{array}{c}\text { BB } \\
(n=103) \\
N(\%)\end{array}$ & $\begin{array}{c}\text { All-Other } \\
\left(\begin{array}{c}n=48) \\
N(\%)\end{array}\right.\end{array}$ & P-value \\
\hline$<37 \mathrm{w}$ at Delivery & $834(10)$ & $86(26)$ & $27(26)$ & $22(46)$ & $<0.001$ \\
\hline No. Living Children & & & & & 0.205 \\
\hline$\geq 3$ & $1869(22)$ & $82(25)$ & $26(25)$ & $13(27)$ & \\
\hline$\leq 2$ & $6798(78)$ & $239(73)$ & $77(75)$ & $34(71)$ & \\
\hline Parity & & & & & 0.346 \\
\hline 0 & $34(0.6)$ & $0(0)$ & $0(0)$ & $0(0)$ & \\
\hline 1 & $2128(39)$ & $70(34)$ & $23(34)$ & $10(31)$ & \\
\hline 2 & $1946(360$ & $72(35)$ & $25(37)$ & $12(38)$ & \\
\hline 3 & $1326(24)$ & $66(32)$ & $20(29)$ & $10(31)$ & \\
\hline $\mathrm{BMI} \geq 30$ at $\mathrm{EPDS}$ & $2646(30)$ & $218(67)$ & $71(69)$ & $38(79)$ & $<0.001$ \\
\hline Tobacco Use & & & & & 0.003 \\
\hline Never & $6807(78)$ & $232(71)$ & $70(68)$ & $30(62)$ & \\
\hline Quit & $1582(18)$ & $74(23)$ & $29(28)$ & $17(35)$ & \\
\hline Current Use & $309(4)$ & $17(5)$ & $4(4)$ & $1(2)$ & \\
\hline $\begin{array}{c}\text { Chronic Medical } \\
\text { Condition }\end{array}$ & $1504(17)$ & $113(35)$ & $46(45)$ & $25(52)$ & $<0.001$ \\
\hline Diabetes & $1050(12)$ & $102(31)$ & $40(39)$ & $24(50)$ & $<0.001$ \\
\hline Thyroid Disease & $595(7)$ & $25(8)$ & $16(15)$ & $8(17)$ & $<0.001$ \\
\hline Renal Disease & $21(0.2)$ & $1(0.3)$ & $1(1)$ & $0(0)$ & 0.350 \\
\hline Corticosteroid & $104(1)$ & $4(1)$ & $4(4)$ & $5(10)$ & $<0.001$ \\
\hline Thyroid Medication & $601(7)$ & $16(5)$ & $18(17)$ & $5(10)$ & $<0.001$ \\
\hline Benzodiazepine & $58(0.7)$ & $4(1)$ & $2(2)$ & $1(2)$ & 0.124 \\
\hline Antidepressant & $366(4)$ & $26(8)$ & $6(6)$ & $6(12)$ & $<0.001$ \\
\hline Lifetime Antidepressant & $1283(15)$ & $72(22)$ & $29(28)$ & $14(29)$ & $<0.001$ \\
\hline
\end{tabular}

BB: $\beta$-blocker, GA: Gestational Age, EPDS: Edinburgh Postnatal Depression Scale, BMI: Body Mass Index.

observational study reported a particularly high incidence of depression in a cohort of patients using propranolol, a highly lipophilic BB, for antiarrhythmic therapy. Follow-up studies detected a significantly higher use of anti-depressants in patients taking BBs compared to patients taking other anti-hypertensives [25], and an increased relative risk of 2.6 of antidepressant prescriptions subsequent to BB use [10]. These studies notably found that the youngest BB users (aged 20 44) had the highest rates of anti-depressant use. More recently, several randomized controlled trials evaluating depression as a secondary outcome of BB use have reported a significant increased risk of fatigue, though a lack of association with depression [26]. Conversely, Liu and colleagues' prospective study revealed 
Table 3. Logistic regression results.

\begin{tabular}{ccccccc}
\hline Outcome & \multicolumn{5}{c}{ Comparison Groups } \\
\hline & $\begin{array}{c}\text { Hypertensive vs } \\
\text { Normotensive }\end{array}$ & \multicolumn{2}{c}{ BB vs No-Med } & \multicolumn{2}{c}{ All-Othervs No-Med } \\
\cline { 2 - 7 } & $\begin{array}{c}\text { OR } \\
(95 \% \mathrm{CI})\end{array}$ & $\begin{array}{c}\mathrm{aOR}^{*} \\
(95 \% \mathrm{CI})\end{array}$ & $\begin{array}{c}\text { OR } \\
(95 \% \mathrm{CI})\end{array}$ & $\begin{array}{c}\text { aOR+ } \\
(95 \% \mathrm{CI})\end{array}$ & $\begin{array}{c}\text { OR } \\
(95 \% \mathrm{CI})\end{array}$ & $\begin{array}{c}\text { aOR+ } \\
(95 \% \mathrm{CI})\end{array}$ \\
\hline \multirow{2}{*}{ EPDS $\geq 10$} & 1.97 & 1.61 & 1.49 & 1.22 & 1.21 & 1.42 \\
& $(1.57-2.49)$ & $(1.17-2.21)$ & $(0.82-2.71)$ & $(0.56-2.63)$ & $(0.60-2.46)$ & $(0.57-3.54)$ \\
Self-harm & 1.98 & 1.76 & 1.00 & 0.84 & 1.07 & 1.04 \\
& $(1.32-2.95)$ & $(1.05-2.95)$ & $(0.39-2.59)$ & $(0.32-2.21)$ & $(0.31-3.78)$ & $(0.29-3.74)$ \\
\hline
\end{tabular}

BB: $\beta$-blocker, EPDS: Edinburgh Postnatal Depression Scale, OR: Unadjusted Odds Ratio, aOR: Adjusted Odds Ratio, CI: Confidence Interval. Significant associations are displayed in bold. ${ }^{\star}$ Models adjusted for EPDS $\geq 10$, teen, race-ethnicity, partner status, health insurance, parity, BMI, benzodiazepine use, tobacco use, antidepressant use, and lifetime antidepressant. Self-harm, teen, race-ethnicity, partner status, health insurance, parity, antidepressant use, and lifetime antidepressant. +Models adjusted for EPDS $\geq 10$, teen, race-ethnicity, partner status, health insurance, parity, BMI, benzodiazepine use, antidepressant use, and lifetime antidepressant. Self-harm, teen, race-ethnicity, partner status, health insurance.

worsened depressive symptoms in congestive heart failure patients treated with metoprolol [12]. These studies notably consisted of relatively elderly and predominately male subjects with heart disease. Furthermore, not all studies evaluated depression through validated questionnaires or interviews. Recently, Ringoir and colleagues conducted a cross-sectional study evaluating depression and $\mathrm{BB}$ use in 573 primary care hypertension patients without prior myocardial infarction or heart failure [9]. This group evaluated depression through a structured interview that included the validated Patient Health Questionnaire 9 (PHQ-9). The authors found that lipophilic BB use is significantly independently associated with depression.

Mechanisms have been proposed to explain the biologic plausibility of how BBs may be associated with depression [13]. $\beta$-Adrenergic receptors are found within the brain. $\beta$-Blockers have also been found to signal through serotonergic receptors in the brain, receptors that directly influence mood. Additionally, BBs may act non-specifically to influence mood through cellular membrane stabilization or via peripheral nervous system changes.

Labetalol is a widely used BB during pregnancy, owing to its long track record of safety in pregnancy [5] [27]. Oral labetalol has a 1:3 $\alpha$ - to $\beta$-blockade ratio [28]. Agents with $\alpha$-blocking properties likely preserve uteroplacental blood flow and are preferred to selective BBs, which are associated with fetal growth restriction and small for gestational age neonates [29]. In many studies revealing a positive association between BB use and depression, a common thread seems to be the association with highly lipophilic $\beta$-antagonists. Labetalol has low lipid solubility [30], possibly helping to explain the negative association between BBs and depression in our study. Notably, while labetalol is a first-line antihypertensive during pregnancy, it is not widely used in the general population.

Limitations of our study include its retrospective design, which limits cause and effect determinations. Additionally, medication use was identified from prescribing information. Compliance with medication use was not indepen- 
dently confirmed, and length of medication use could not be ascertained. Our analysis relies on the accuracy of the electronic health record (EHR). Prior evaluation of our integrated health system's EHR demonstrated high concordance with chart review, and particularly high concordance with hypertension diagnoses, supporting the accuracy of our EHR [31]. To insure that we captured women who met criteria for gestational hypertensive disorders, we utilized both ICD-9-CMcodes and blood pressure criteria over the course of pregnancy. This strategy allowed for careful identification of women with hypertensive disease, though it limits subgroup analyses by type of hypertension. Strengths of our study include the large sample size, the ability to categorize women by hypertension and BB use, and the validated EPDS tool utilized to assess for depression risk. Additionally, our 5\% incidence of hypertension in pregnancy is in line with United States population estimates of antepartum hypertension [6].

Combating peripartum depression is paramount to a healthy pregnancy. Reducing depression begins with effective screening. Our findings here emphasize the need for extra attention to depression evaluation in those women with hypertension during pregnancy. In addition to universal depression screening during the perinatal period, as recommended by the American College of Obstetricians and Gynecologists [3], additional depression screening in women with antepartum hypertension may be appropriate. Prospective studies are needed to help elucidate causality. Further investigations are also needed to define more precise benefits of depression screening along with optimum management strategies in screen-positive women. Reassuringly, BB treatment of hypertension does not appear to be associated with depression risk.

\section{Conflicts of Interest}

The authors declare no conflicts of interest regarding the publication of this paper.

\section{References}

[1] Bennett, H.A., Einarson, A., Taddio, A., Koren, G. and Einarson, T.R. (2004) Prevalence of Depression during Pregnancy: Systematic Review. Obstetrics and Gynecology, 103, 698-709. https://doi.org/10.1097/01.AOG.0000116689.75396.5f

[2] Stewart, D.E. (2011) Clinical Practice. Depression during Pregnancy. The New England Journal of Medicine, 365, 1605-1611. https://doi.org/10.1056/NEJMcp1102730

[3] American College of Obstetricians and Gynecologists, Committee on Obstetric Practice (2015) Committee Opinion No. 630: Screening for Perinatal Depression. Obstetrics and Gynecology, 125, 1268-1271. https://doi.org/10.1097/01.AOG.0000465192.34779.dc

[4] Lai, C., Coulter, S.A. and Woodruff, A. (2017) Hypertension and Pregnancy. Texas Heart Institute Journal, 44, 350-351. https://doi.org/10.14503/THIJ-17-6359

[5] American College of Obstetricians and Gynecologists, Task Force on Hypertension in Pregnancy (2013) Hypertension in pregnancy. Report of the American College of Obstetricians and Gynecologists' Task Force on Hypertension in Pregnancy. Obstetrics and Gynecology, 122, 1122-1131. 
[6] Kuklina, E.V., Ayala, C. and Callaghan, W.M. (2009) Hypertensive Disorders and Severe Obstetric Morbidity in the United States. Obstetrics \& Gynecology, 113, 1299-1306. https://doi.org/10.1097/AOG.0b013e3181a45b25

[7] Katon, W.J., Russo, J.E., Melville, J.L., Katon, J.G. and Gavin, A.R. (2012) Depression in Pregnancy Is Associated with Preexisting but Not Pregnancy-Induced Hypertension. General Hospital Psychiatry, 34, 9-16. https://doi.org/10.1016/j.genhosppsych.2011.09.018

[8] Kurki, T., Hiilesmaa, V., Raitasalo, R., Mattila, H. and Ylikorkala, O. (2000) Depression and Anxiety in Early Pregnancy and Risk for Preeclampsia. Obstetrics \& Gynecology, 95, 487-490.

[9] Ringoir, L., Pedersen, S.S., Widdershoven, J.W., Pouwer, F., Keyzer, J.M., Romeijnders, A.C. and Pop, V.J. (2014) Beta-Blockers and Depression in Elderly Hypertension Patients in Primary Care. Family Medicine, 46, 447-453.

[10] Thiessen, B.Q., Wallace, S.M., Blackburn, J.L., Wilson, T.W. and Bergman, U. (1990) Increased Prescribing of Antidepressants Subsequent to Beta-Blocker Therapy. Archives of Internal Medicine, 150, 2286-2290. https://doi.org/10.1001/archinte.1990.00390220044009

[11] Waal, H.J. (1967) Propranolol-Induced Depression. British Medical Journal, 2, 50. https://doi.org/10.1136/bmj.2.5543.50

[12] Liu, X., Lou, X., Cheng, X. and Meng, Y. (2017) Impact of Metoprolol Treatment on Mental Status of Chronic Heart Failure Patients with Neuropsychiatric Disorders. Drug Design, Development and Therapy, 11, 305-312. https://doi.org/10.2147/DDDT.S124497

[13] Koella, W.P. (1985) CNS-Related (Side-)Effects of Beta-Blockers with Special Reference to Mechanisms of Action. European Journal of Clinical Pharmacology, 28, 55-63. https://doi.org/10.1007/BF00543711

[14] Murray, L. and Carothers, A.D. (1990) The Validation of the Edinburgh Post-Natal Depression Scale on a Community Sample. The British Journal of Psychiatry, 157, 288-290. https://doi.org/10.1192/bjp.157.2.288

[15] Bergink, V., Kooistra, L., Lambregtse-van den Berg, M.P., Wijnen, H., Bunevicius, R., van Baar, A. and Pop, V. (2011) Validation of the Edinburgh Depression Scale during Pregnancy. Journal of Psychosomatic Research, 70, 385-389. https://doi.org/10.1016/j.jpsychores.2010.07.008

[16] Celano, C.M., Freudenreich, O., Fernandez-Robles, C., Stern, T.A., Caro, M.A. and Huffman, J.C. (2011) Depressogenic Effects of Medications: A Review. Dialogues in Clinical Neuroscience, 13, 109-125.

[17] Igwe, M.N., Uwakwe, R., Ahanotu, C.A., Onyeama, G.M., Bakare, M.O. and Ndukuba, A.C. (2013) Factors Associated with Depression and Suicide among Patients with Diabetes Mellitus and Essential Hypertension in a Nigerian Teaching Hospital. African Health Sciences, 13, 68-77. https://doi.org/10.4314/ahs.v13i1.10

[18] Munhoz, T.N., Nunes, B.P., Wehrmeister, F.C., Santos, I.S. and Matijasevich, A. (2016) A Nationwide Population-Based Study of Depression in Brazil. Journal of Affective Disorders, 192, 226-233. https://doi.org/10.1016/j.jad.2015.12.038

[19] Carney, R.M. and Freedland, K.E. (2008) Depression in Patients with Coronary Heart Disease. American Journal of Medicine, 121, S20-S27. https://doi.org/10.1016/j.amjmed.2008.09.010

[20] Miller, A.H. and Raison, C.L. (2016) The Role of Inflammation in Depression: From Evolutionary Imperative to Modern Treatment Target. Nature Reviews Immunology, 16, 22-34. https://doi.org/10.1038/nri.2015.5 
[21] Aschbacher, K., Epel, E., Wolkowitz, O.M., Prather, A.A., Puterman, E. and Dhabhar, F.S. (2012) Maintenance of a Positive Outlook during Acute Stress Protects against Pro-Inflammatory Reactivity and Future Depressive Symptoms. Brain, Behavior, and Immunity, 26, 346-352. https://doi.org/10.1016/j.bbi.2011.10.010

[22] Buglione-Corbett, R., Deligiannidis, K.M., Leung, K., Zhang, N., Lee, M., Rosal, M.C. and Moore Simas, T.A. (2018) Expression of Inflammatory Markers in Women with Perinatal Depressive Symptoms. Archives of Women's Mental Health.

[23] Simpson, W., Steiner, M., Coote, M. and Frey, B.N. (2016) Relationship between Inflammatory Biomarkers and Depressive Symptoms during Late Pregnancy and the Early Postpartum Period: A Longitudinal Study. Revista Brasileira de Psiquiatria, 38, 190-196. https://doi.org/10.1590/1516-4446-2015-1899

[24] Hallas, J. (1996) Evidence of Depression Provoked by Cardiovascular Medication: A Prescription Sequence Symmetry Analysis. Epidemiology, 7, 478-484. https://doi.org/10.1097/00001648-199609000-00005

[25] Avorn, J., Everitt, D.E. and Weiss, S. (1986) Increased Antidepressant Use in Patients Prescribed Beta-Blockers. JAMA, 255, 357-360. https://doi.org/10.1001/jama.1986.03370030077031

[26] Ko, D.T., Hebert, P.R., Coffey, C.S., Sedrakyan, A., Curtis, J.P. and Krumholz, H.M. (2002) Beta-Blocker Therapy and Symptoms of Depression, Fatigue, and Sexual Dysfunction. JAMA, 288, 351-357. https://doi.org/10.1001/jama.288.3.351

[27] Fitton, C.A., Steiner, M.F.C., Aucott, L., Pell, J.P., Mackay, D.F., Fleming, M. and McLay, J.S. (2017) In-Utero Exposure to Antihypertensive Medication and Neonatal and Child Health Outcomes: A Systematic Review. Journal of Hypertension, 35, 2123-2137. https://doi.org/10.1097/HJH.0000000000001456

[28] Richards, D.A., Tuckman, J. and Prichard, B.N. (1976) Assessment of Alpha- and Beta-Adrenoceptor Blocking Actions of Labetalol. British Journal of Clinical Pharmacology, 3, 849-855. https://doi.org/10.1111/j.1365-2125.1976.tb00637.x

[29] Lydakis, C., Lip, G.Y., Beevers, M. and Beevers, D.G. (1999) Atenolol and Fetal Growth in Pregnancies Complicated by Hypertension. American Journal of Hypertension, 12, 541-547. https://doi.org/10.1016/S0895-7061(99)00031-X

[30] Poirier, L. and Tobe, S.W. (2014) Contemporary Use of Beta-Blockers: Clinical Relevance of Subclassification. Canadian Journal of Cardiology, 30, S9-S15. https://doi.org/10.1016/j.cjca.2013.12.001

[31] Straub, H., Adams, M. and Silver, R.K. (2014) Can an Electronic Health Record System Be Used for Preconception Health Optimization? Maternal and Child Health Journal, 18, 2134-2140. https://doi.org/10.1007/s10995-014-1461-8 